\title{
A Noble Classification Framework for Data Glove Classification of a Large Number of Hand Movements
}

\author{
Yuhuang Zheng (D) $^{1,2}$ \\ ${ }^{1}$ Academic Affairs Office, Guangdong University of Education, Guangzhou 510303, China \\ ${ }^{2}$ Guangdong Province Key Laboratory of Precision Equipment and Manufacturing Technology, Guangzhou 510641, China \\ Correspondence should be addressed to Yuhuang Zheng; zhyhaa@126.com
}

Received 28 August 2021; Revised 1 October 2021; Accepted 4 October 2021; Published 27 October 2021

Academic Editor: Yang Li

Copyright (c) 2021 Yuhuang Zheng. This is an open access article distributed under the Creative Commons Attribution License, which permits unrestricted use, distribution, and reproduction in any medium, provided the original work is properly cited.

\begin{abstract}
The recognition of hand movements is an important method for human-computer interaction (HCI) technology, and it is widely used in virtual reality and other HCI areas. While many valuable efforts have been made, efficient ways to capture over 20 types of hand movements with high accuracy by one data glove are still lacking. This paper addresses a new classification framework for 52 hand movements. This classification framework includes the following two parts: the movement detection algorithm and the movement classification algorithm. The fine K-nearest neighbor (Fine KNN) is the core of the movement detection algorithm. The movement classification algorithm is composed of downsampling in data preparation and a new deep learning network named the DBDF network. Bidirectional Long Short-Term Memory (BiLSTM) is the main part of the DBDF network. The results of experiments using the Ninapro DB1 dataset demonstrate that our work can classify more types of hand movements than related algorithms with a precision of $93.15 \%$.
\end{abstract}

\section{Introduction}

As one of the hotspots in the research field of humancomputer interaction (HCI) technology, hand movement recognition has been deeply studied by researchers and has been widely used in virtual reality, artificial intelligence, and other HCI areas. With the popularization and application of advanced sensors, hand movement recognition based on contact sensors has developed rapidly, especially data glove technology. Data gloves can more intuitively obtain the three-dimensional spatial information of hand posture by using multiple sensors and are not constrained by the surrounding environment.

In the virtual reality scene, the user's different intentions can be judged by hand movement recognition, which facilitates the user's manipulation of objects in the virtual scene and improves the user's immersive experience. In daily life, the emergence of smart homes also provides more application fields for hand movement recognition. Especially in virtual reality training scenes, users need a simpler and faster operation and adapt to reality, which makes the traditional HCI mode of mouse keyboards gradually replaced by data gloves. Data glove technology allows users to manipulate objects in the training scene only through gesture changes so that users have a realistic immersive experience. Hand movement recognition has great potential for development in the future.

According to whether there is a time series of actions, hand movement recognition is divided into the following two types: static and dynamic recognition. Static recognition refers to recognition and classification without time series, focusing only on changes in hand spatial features, such as hand shape, contour, and center of gravity. However, the system often needs continuous instruction rather than a single, on-off instruction, which makes the static gesture unable to meet the needs of HCI. Dynamic hand movement recognition can be understood as the recognition of static gesture combinations in a period of time series, which not 
only provides users with better fluency but also compensates for the defects of static gesture recognition in humancomputer interactions.

At the same time, according to different methods of collecting gesture interaction information, gesture recognition can be divided into gesture recognition based on noncontact sensors and contact sensors. A camera is one kind of typical noncontact sensor. An image or video of hand movement is collected using a camera, and the processed gesture information is recognized synthetically by gesture detection, tracking, positioning, and other methods. The camera-based hand movement recognition method has been widely used in smart homes, but it is greatly affected by the background environment, especially in the case of weak illumination, low camera pixels, and complex and changeable backgrounds. Therefore, this method is not suitable for the application of virtual reality scenes. Hand movement recognition based on contact sensors refers to the recognition method of obtaining hand information through sensors. This method generally refers to hand movement recognition based on data gloves. There are many sensors in the data glove. Through these sensors, we can obtain various hand change data in real time without omission and then extract and classify the features of these data to finally achieve hand movement recognition. Compared with the camera-based hand movement recognition method, this method has faster recognition speed and higher accuracy. At the same time, when users use data gloves for hand movement recognition, they have better comfort and freedom because they do not need to consider the position of the camera.

In the past, due to the slow development of sensor technology, the accuracy of data gloves was not high, and the price was expensive, which made the camera-based hand movement recognition method dominant in the field of gesture recognition. In recent years, with the rapid development of sensor technology, the cost of data gloves has been reduced. The feasibility of hand movement recognition methods based on data gloves in various fields has become increasingly obvious, and people have paid increasing attention to them. Therefore, research on hand movement recognition technology based on data gloves plays an increasingly important role in this recognition field. Nassour et al. [1] proposed a sensory glove and used machine learning algorithms to estimate the angles of the joints in the hand and to identify 15 gestures with an average accuracy of $89.4 \%$. Chen et al. [2] presented a wearable hand rehabilitation system that offers 16 kinds of finger gestures with an accuracy of $93.32 \%$. Pan et al. [3] presented a wireless smart glove that can recognize 10 American Sign Language gestures, and the highest testing classification accuracy of our system is $99.7 \%$. Maitre et al. [4] developed a data glove prototype allowing for the recognition of objects in 8 basic daily activities with an accuracy of 95\%. Lee and Bae [5] proposed a real-time gesture recognition system that uses a data glove to classify 11 gestures, and the recognition result showed 100\% accuracy. Ayodele et al. [6] proposed the use of convolutional neural networks on 6 grasp classifications using a piezoresistive data glove, and the average classification accuracy of the convolutional neural networks (CNN) algorithm was $88.27 \%$ and $75.73 \%$ in the object-seen and object-unseen scenarios, respectively. Chauhan et al. [7] presented grasp prediction algorithms that can be used for naturalistic, synergistic control of exoskeleton gloves for 5 activities, and an average accuracy of approximately $75 \%$ was achieved. In [8], an automatic recognition algorithm to identify hand movements using surface electromyogram signals was proposed with an average accuracy of $93.53 \%$ on Ninapro DB5 (17 gestures). In [9], a prototype of a data glove was proposed that performs well object recognition during 13 basic daily activities, and the accuracy was almost $93 \%$. Huang et al. [10] used a prefabricated data glove to monitor the bending angle of the finger joint in real time and then realized the recognition of 9 Chinese Sign Language (CSL) words in which the accuracy reached $98.3 \%$. A novel deep learning framework based on the graph convolutional neural networks (GCNs-Net) was presented in [11] and GCNs-Net achieved the highest averaged accuracy $96.24 \%$ at the subject level on the High Gamma dataset (4 movements). In [12], an attention-based BiLSTM-GCN was proposed to accurately classify four-class electroencephalogram motor imagery tasks and showed prediction based on individual training with $98.81 \%$ accuracy.

Although many scholars have made remarkable development in the field of hand movements recognition, there is still a need to improve the classification accuracy of multiple hand movements [13]. The recognition of multiple hand movements is challenging because the accuracy typically decreases obviously with the addition of more hand movements [14]. However, to the best of our knowledge, there are few actions in the existing research that can be recognized with high precision when only using data gloves. In the above research using data gloves, the average accuracy exceeds $90 \%$, but the number of hand movements does not exceed 20 types. Towards accurate and more hand movements recognition from raw signals of data gloves, a new classification framework should be constructed.

Therefore, in this paper, we propose applying the fine K-nearest neighbor (Fine KNN) and Bidirectional Long Short-Term Memory (BiLSTM) in classifying a larger Ninapro DB1 dataset including 52 movements with 22 sensor measurements of one data glove. The classification framework is divided into two steps. First, a Fine KNN classifier is used to obtain the sensor sequence of a hand movement over a period and determines whether the sequence is a resting state or movement state. Second, a classification model, named the DBDF network, is proposed to recognize 52 movements for the movement state sensor sequence. The structure of this classification framework is shown in Figure 1. Our results show that the classification framework achieved the recognition of the 52 hand movements in Ninapro DB1 only by one data glove with an accuracy of $93.15 \%$. 


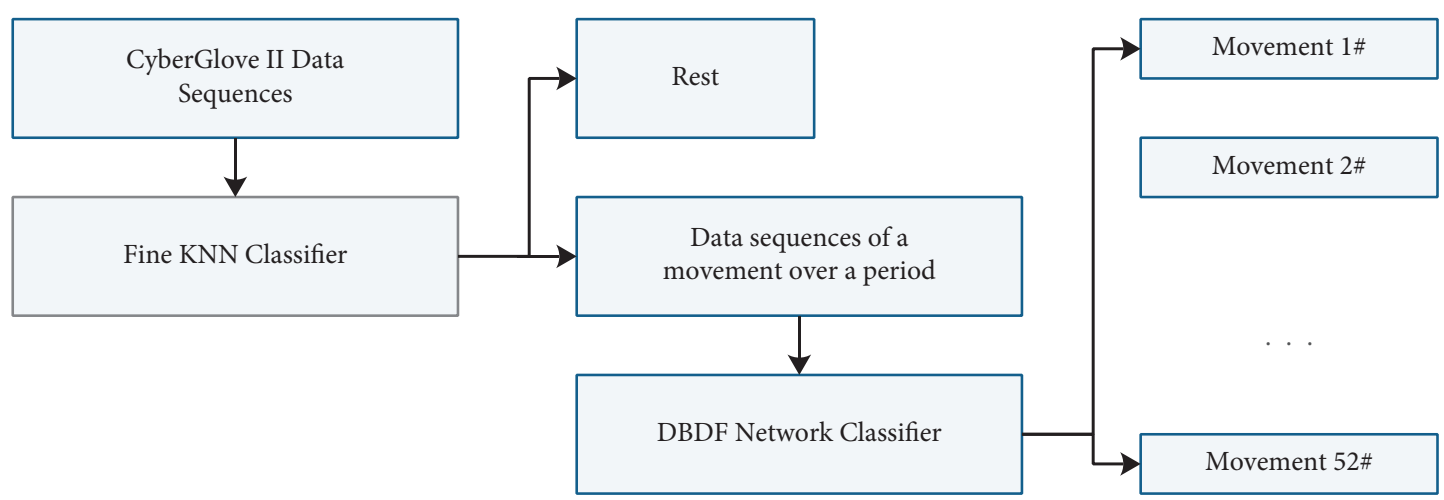

FIgURE 1: The structure of this classification framework.

The main contributions of this work can be summarized under three headings:

(1) A novel classification framework is introduced to detect 52 hand movements while just cooperating with data gloves.

(2) The hand movement classification algorithm on the benchmark datasets of Ninapro DB1 performs better than the existing state-of-the-art algorithms in recognizing the number of hand movements and maintains high classification accuracy.

(3) The hand movement classification algorithm has good scalability when the input size grows larger. Data preparation involves the downsampling technology to rescale input variables before training the DBDF network and the downsampling technology improves neural network stability and modeling performance by scaling data.

The structure of the rest of this paper is as follows. Section 2 describes the Ninapro DB1. Section 3 introduces the hand movement detection algorithm based on Fine KNN. The hand movement classification algorithm is reported in Section 4. Sections 5 and 6 illustrate the experimental results and conclusion, respectively.

\section{Ninapro DB 1}

Ninapro $[15,16]$ is a publicly available multimodal database to foster research on robotic hands controlled with artificial intelligence. Ninapro includes electromyography, kinematic, inertial, eye tracking, visual, clinical, and neurocognitive data. Ninapro DB1 includes data from 27 intact subjects. We use hand kinematics data in Ninapro DB1 as our experimental data. In Ninapro DB1, 52 hand movements and the Reset state are listed in Table 1 [16]. Seventy percent of Ninapro DB1 is used as the training set, and $30 \%$ is used as the testing set in the experiment.

Hand kinematics were measured for all subjects using a 22sensor CyberGlove II data glove. CyberGlove II is a motion capture data glove instrumented with joint-angle measurement sensors. It uses proprietary resistive bend-sensing technology to transform hand and finger motions into real-time digital joint-angle data. The number and the corresponding position of each CyberGlove II sensor are shown in Figure 2.

\section{The Movement Detection Algorithm}

3.1. Algorithm Introduction. Sample data of CyberGlove II are represented symbolically as follows:

$s_{j}=\left[s_{i, j}\right]_{i: 1, \ldots, 22}=\left[\begin{array}{llll}s_{1, j} & s_{2, j} & \ldots & s_{22, j}\end{array}\right]^{T}, \quad$ where $j=1,2,3$.

The corresponding movement label is defined as follows:

$$
a l_{j}= \begin{cases}0, & \text { reset, } \\ 1, & \text { movement. }\end{cases}
$$

This window can provide different transition widths for the same $L$, which is something other fixed windows lack:

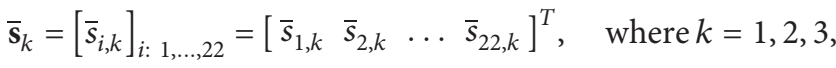

$\bar{s}_{i, k}=\frac{1}{L} \sum_{j=(k-1) L+1}^{k L} s_{i, j}$.

Define $\widetilde{\mathbf{s}}_{k}$ as a subvector extracted from a vector $\overline{\mathbf{s}}_{k}$. The movement label is as follows:

$$
\overline{a l}_{k}=\left\lceil\frac{1}{L} \sum_{j=(k-1) L+1}^{k L} a l_{j}\right\rceil .
$$

Hand movement detection is a classification that classifies the data $\widetilde{\mathbf{s}}_{k}$ between two classes in "0" and " 1 " of $\overline{a l}_{k}$.

Let the training set be $\left(\widetilde{\mathbf{s}}_{1}, \overline{a l}_{1}\right),\left(\widetilde{\mathbf{s}}_{2}, \overline{a l}_{2}\right), \ldots$, where $\widetilde{\mathbf{s}}_{k}$ is an input vector and $\overline{a l}_{k}$ its label. The K-nearest neighbors (KNN) [17] algorithm is a type of supervised machine learning algorithm that can be used for both classification and regression predictive problems. However, it is mainly used for classification predictive problems in industry. KNN is used to train the dataset and classify the dataset based on similarity and distance measures. In this paper, Fine KNN is a classifier in which the nearest neighbors are determined based on Euclidean distance, which makes finely detailed 
Table 1: Ninapro DB1.

\begin{tabular}{|c|c|c|}
\hline Label & Movement & Exercise \\
\hline 0 & Rest & $A, B, C$ \\
\hline 1 & Index flexion & A \\
\hline 2 & Index extension & $\mathrm{A}$ \\
\hline 3 & Middle flexion & $\mathrm{A}$ \\
\hline 4 & Middle extension & $\mathrm{A}$ \\
\hline 5 & Ring flexion & A \\
\hline 6 & Ring extension & $\mathrm{A}$ \\
\hline 7 & Little finger flexion & $\mathrm{A}$ \\
\hline 8 & Little finger extension & $\mathrm{A}$ \\
\hline 9 & Thumb adduction & $\mathrm{A}$ \\
\hline 10 & Thumb abduction & A \\
\hline 11 & Thumb flexion & A \\
\hline 12 & Thumb extension & A \\
\hline 13 & Thumb up & $\mathrm{B}$ \\
\hline 14 & Extension of index and middle, flexion of the others & $\mathrm{B}$ \\
\hline 15 & Flexion of ring and little finger, extension of the others & $\mathrm{B}$ \\
\hline 16 & Thumb opposing base of little finger & $\mathrm{B}$ \\
\hline 17 & Abduction of all fingers & $\mathrm{B}$ \\
\hline 18 & Fingers flexed together in fist & $\mathrm{B}$ \\
\hline 19 & Pointing index & $\mathrm{B}$ \\
\hline 20 & Adduction of extended fingers & $\mathrm{B}$ \\
\hline 21 & Wrist supination (axis: middle finger) & $\mathrm{B}$ \\
\hline 22 & Wrist pronation (axis: middle finger) & $\mathrm{B}$ \\
\hline 23 & Wrist supination (axis: little finger) & $\mathrm{B}$ \\
\hline 24 & Wrist pronation (axis: little finger) & $\mathrm{B}$ \\
\hline 25 & Wrist flexion & $\mathrm{B}$ \\
\hline 26 & Wrist extension & $\mathrm{B}$ \\
\hline 27 & Wrist radial deviation & $\mathrm{B}$ \\
\hline 28 & Wrist ulnar deviation & $\mathrm{B}$ \\
\hline 29 & Wrist extension with closed hand & $\mathrm{B}$ \\
\hline 30 & Large diameter grasp & $\mathrm{C}$ \\
\hline 31 & Small diameter grasp (power grip) & $\mathrm{C}$ \\
\hline 32 & Fixed hook grasp & $\mathrm{C}$ \\
\hline 33 & Index finger extension grasp & $\mathrm{C}$ \\
\hline 34 & Medium wrap & $\mathrm{C}$ \\
\hline 35 & Ring grasp & $\mathrm{C}$ \\
\hline 36 & Prismatic four fingers grasp & $\mathrm{C}$ \\
\hline 37 & Stick grasp & $\mathrm{C}$ \\
\hline 38 & Writing tripod grasp & $\mathrm{C}$ \\
\hline 39 & Power sphere grasp & $\mathrm{C}$ \\
\hline 40 & Three finger sphere grasp & $\mathrm{C}$ \\
\hline 41 & Precision sphere grasp & $\mathrm{C}$ \\
\hline 42 & Tripod grasp & $\mathrm{C}$ \\
\hline 43 & Prismatic pinch grasp & $\mathrm{C}$ \\
\hline 44 & Tip pinch grasp & $\mathrm{C}$ \\
\hline 45 & Quadrupod grasp & $\mathrm{C}$ \\
\hline 46 & Lateral grasp & $\mathrm{C}$ \\
\hline 47 & Parallel extension grasp & $\mathrm{C}$ \\
\hline 48 & Extension type grasp & $\mathrm{C}$ \\
\hline 49 & Power disk grasp & $\mathrm{C}$ \\
\hline 50 & Open a bottle with a tripod grasp & $\mathrm{C}$ \\
\hline 51 & Turn a screw (grasp the screwdriver with a stick grasp) & $\mathrm{C}$ \\
\hline 52 & Cut something (grasp the knife with an index finger extension grasp) & $\mathrm{C}$ \\
\hline
\end{tabular}

distinctions among classes with the number of neighbors set to 1 because of just two classes in this movement label. Define $\overline{a l}_{k}^{*}$ as the Fine KNN classifier prediction of $\left(\widetilde{\mathbf{s}}_{k}, \overline{a l}_{k}\right)$.
If $\quad \overline{a l}_{k}=\overline{a l}_{k+1}=\cdots=\overline{a l}_{k+r}, \overline{a l}_{k} \neq \overline{a l}_{k-1}, \overline{a l}_{k+r} \neq \overline{a l}_{k+r+1}$, $r>L$, then a whole movement period is composed of the measurement of CyberGlove II: 


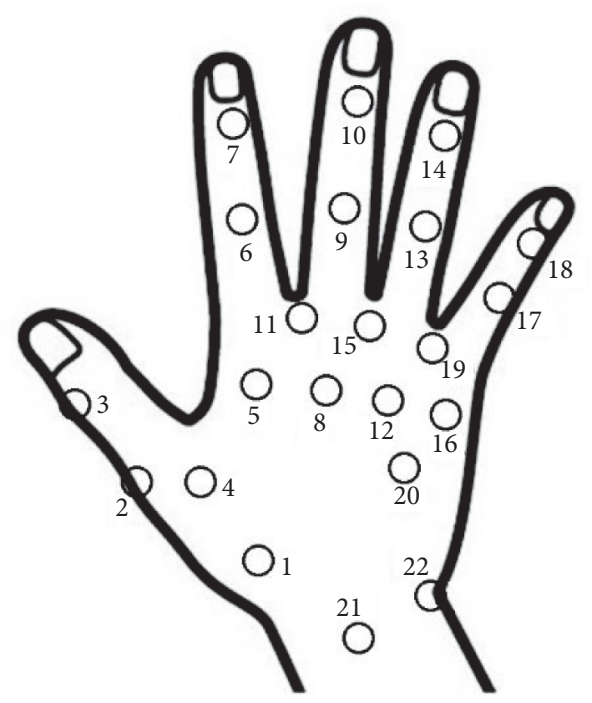

FIgure 2: The corresponding position of each CyberGlove II sensor.

$x[(k+r) L]=\left\{\mathbf{s}_{j+1}, \ldots, \mathbf{s}_{j+L}, \ldots, \mathbf{s}_{j+(k+r-1) L+1}, \ldots, \mathbf{s}_{j+(k+r) L}\right\}$.

3.2. Algorithm Effect Analysis. The thumb is the most important part of the hand. The thumb has only two bones, so it is obviously shorter and plays a very important role, which cannot be done by other fingers in physiology. In this paper, we select the number of sensors $(1,2,3,4,5,6,9,13,17)$ and $\widetilde{\mathbf{s}}_{k}$ is defined as follows:

$$
\begin{array}{r}
\widetilde{\mathbf{s}}_{k}=\left[\begin{array}{lllllllll}
\bar{s}_{1, k} & \bar{s}_{2, k} & \bar{s}_{3, k} & \bar{s}_{4, k} & \bar{s}_{5, k} & \bar{s}_{6, k} & \bar{s}_{9, k} & \bar{s}_{13, k} & \bar{s}_{17, k}
\end{array}\right]^{T}, \\
k=1,2,3, \ldots, L=10 .
\end{array}
$$

As a result, in Table 2, the Fine KNN by the number of sensors $(1,2,3,4,5,6,9,13,17)$ achieved the highest accuracy. The Fine KNN achieved the highest $99.4 \%$ completeness of the test dataset among other numbers of sensors. The Fine KNN classifier is selected for the optimized classified model. Figure 3 shows the confusion matrix of Fine KNN. This classification model can effectively obtain a whole movement period [18].

\section{The Movements Classification Algorithm}

The model presented in Section 3 can detect only the hand movement state and its movement period, but it is necessary to propose a new model to recognize 52 hand movements. Hand movement recognition is mainly classified and recognized according to the characteristics of hand movements, and the feature extraction of hand movements is based on the data in the training sample dataset. Therefore, the quality of the data in the training sample dataset directly affects the subsequent hand movement recognition.
TABLE 2: Accuracy with different numbers of sensors.

\begin{tabular}{lc}
\hline Numbers of sensors & $\begin{array}{r}\text { Accuracy of } \overline{a l}_{k}^{*} \text { prediction in } \\
\text { test set }(\%)\end{array}$ \\
\hline $1,2,3,4,5$ & 91.1 \\
$3,6,9,13,17$ & 90.1 \\
$1,2,3,4,5,6,9,13,17$ & 99.4 \\
$3,7,10,14,18$ & 89.9 \\
$2,3,6,7,9,10,13,14,17,18$ & 98.2 \\
\hline
\end{tabular}

\begin{tabular}{c|c|c|c|}
\multicolumn{3}{c}{} & \multicolumn{2}{c}{ Predicted Class } \\
\cline { 2 - 4 } True & & 0 (Rest) & 1 (Movement) \\
\cline { 2 - 4 } Class & 713042 & 3353 \\
\cline { 2 - 4 } & 0 (Rest) & 3930 & 534994 \\
\cline { 2 - 4 } & 1 (Movement) & $99.4 \%$ &
\end{tabular}

Figure 3: The confusion matrix of Fine KNN.

4.1. Data Preparation. In Ninapro DB1, each hand movement period is not the same; the shortest sample is approximately 200 records, and the longest sample is nearly 1000 records. It should process records of these movements to make the length of every movement sample close. Downsampling technology is used to process these records. Downsampling, which is also sometimes called decimation, reduces the sampling rate. The idea of downsampling is to remove records from the signal while maintaining its length with respect to time.

The sensor data rate of CyberGlove II is 90 records/second typical. This means that the sampling frequency of CyberGlove II is $f_{s}=90 \mathrm{~Hz}$. The shortest sample time of a whole hand movement is $200 / 90=2.2$ seconds. The longest sample time was approximately $1000 / 90=11$ seconds. This means that the frequency $f$ range of a whole hand movement is $0.1 \mathrm{~Hz}$ to $0.45 \mathrm{~Hz}$. If the downsampling algorithm is used to change the number of samples for every movement between 100 and 200 , the new sampling frequency $f_{s}^{\prime}$ will change from $9 \mathrm{~Hz}$ (1000 records) to $90 \mathrm{~Hz}$ (200 records), and $f_{s}^{\prime}>2 f$ will be conducted. According to the Nyquist theorem, new samples processed by downsampling enable a complete reconstruction of the corresponding original samples.

Consider downsampling a labeled signal $x[n]$ of length $N$ and reducing the number of samples $N$ by a factor of $n_{s}$, where $n_{s}$ is a divisor of $N$. A new signal is represented as follows:

$$
y[m]=x\left[m n_{s}\right], 0 \leq m<\frac{N}{n_{s}}, \frac{N}{n_{s}} \in[100,200],
$$

where $y[m]$ is the input of the hand movement classifier below.

4.2. DBDF Network. Essentially, hand movement recognition is a sequence classification, which is a predictive modeling problem where the sensor sequence of CyberGlove II and the task is to predict a hand movement category for 
the sequence. What makes this problem difficult is that the sensor sequences of CyberGlove II can vary in length. Although the sensor sequences have been shortened by downsampling, the classifier is required to learn the long term in the input sequence.

Recently, deep learning methods have been shown to provide state-of-the-art results on challenging hand movement recognition tasks with little or no data feature engineering instead of using feature learning on raw data.

The LSTM (Long Short-Term Memory) [19] network model is a recurrent neural network that can learn and memorize long sequences of input data. BiLSTM [20] is an extension of traditional LSTM, which can improve the performance of the model on sequence classification problems. In this problem, where all time steps of the input sequence are available, BiLSTM trains two LSTMs on the input sequence instead of one. The first LSTM is on the input sequence, and the second is on the reverse copy of the input sequence. This can provide additional context for the network to understand the problem faster and more comprehensively.

The BiLSTM network can support multiple parallel sequences of input data, such as each sensor of the CyberGlove II data. The BiLSTM network learns to extract features from sequences of observations and how to map the internal features to different movement categories.

The benefit of using BiLSTM for sequence classification is that they can learn from the raw time series data directly and in turn do not require domain expertise to manually engineer input features. The BiLSTM network can learn an internal representation of the time series data and ideally achieve comparable performance to models fit on a version of the dataset with engineered features. BiLSTM is an effective solution that obtains access to both preceding and succeeding information by involving two separate hidden layers with opposite information flow directions. The traditional BiLSTM classification network usually uses the final state for classification.

Since sensor records show the time dependence of dynamic CyberGlove II sensor data, to better classify the sensor time series of CyberGlove II and further improve the classification accuracy of the sensor sequences, a classification network composed of double BiLSTM layers (bilstmLayer) and double fully connected layers (fullyConnectedLayer) is proposed, called the DBDF network. The association relationship between a sequence of CyberGlove II sensors and the forward and backward association relationship of the sequence itself can be fully extracted by the DBDF network. It has the advantage of an easy training process, and the computational complexity is reduced [21].

A DBDF network is designed to classify the experiment in Section 3, and the structure of this model is shown in Figure 4. The BiLSTM cell is the core of the DBDF network. The joint-angle measurement data of CyberGlove II are used as the input of the DBDF network. The outputs of each repeating BiLSTM cell are concatenated into a dense layer for further prediction. In the DBDF network, two fully connected layers are added after two bilstmLayers. In this network, the numbers of hidden units in bilstmLayers \#1 and $\# 2$ are 350 , and the output sizes for fullyConnectedLayer \#1 and fullyConnectedLayer \#2 are 200 and 52, respectively. The two bilstmLayers extract features from the sensor sequences, concatenate them together, and map them to two fullyConnectedLayers. The combination of three bilstmLayers increases the amount of data, which can then improve the accuracy and stability of classification [22].

\section{Experiments and Analysis}

5.1. Experimental Results. The DBDF network training and testing are completed under the deep learning framework of MATLAB 2020B with Intel i7-8750h@ @2.20 GHz CPU, 32 GB memory, Nvidia RTX 2070 GPU. Training and testing of the DBDF network are accelerated by the GPU. The RMSProp optimizer is used to train the network, and the network weights and biases are updated continuously. The learning rate was reduced by a factor of 0.1 every 10 epochs. To set the maximum number of epochs for training to 30 , a minibatch with 128 observations was used at each iteration. The RMSProp optimizer algorithm is used to monitor the training error "MSE" to update the model parameters continuously. The learning rate is 0.0001 , and the maximum training epoch is 30 .

The training process usually takes approximately 6 minutes on a single GPU, and the network achieves a training accuracy of $99.5 \%$ and a validation accuracy of $93 \%$. The confusion matrix is used in the performance analysis of classification algorithms. The testing result is shown in the confusion matrix chart in Figure 5, and the accuracy for 52 hand movements is listed in Table 3 . The prediction accuracy of movements $2,3,4,7,10,15,16,19$ can achieve $100 \%$, and the accuracy of movements $43,9,52,44,21$ is lower than $80 \%$. Improving the recognition accuracy of these movements is the focus of the future.

We compare the number of movements and classification accuracy of the proposed methodology with the existing hand movement recognition algorithms in Table 4. We conclude that the proposed algorithm of the DBDF network performs better than the existing state-of-the-art algorithms in recognizing the number of hand movements and maintains high classification accuracy.

\subsection{Classification Network Structure and Downsampling.} The deep learning model in the hand movement classification algorithm is composed of double BiLSTM layers and double fully connected layers in Section 4. We also consider changing the number of bilstmLayers and fullyConnectedLayers. The experimental results are shown in Table 5. As the number of layers increases, the training time of the classification network increases. The DBDF network outperforms other classification networks in Table 5.

In this research, downsampling is very important in data preparation. Downsampling the sensor sequences will save considerable time when training the network, and the validation accuracy of classification will increase. In the case of these variable-length sequence problems, data should be transformed such that each sequence has the same length as 


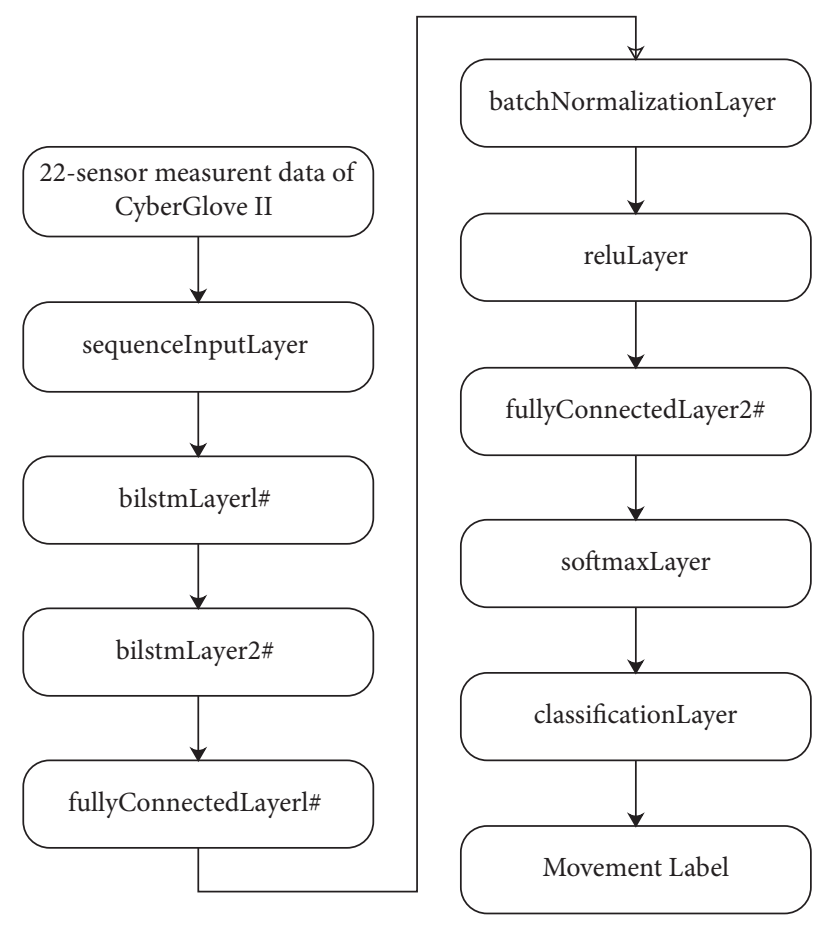

FIgURE 4: The structure of the DBDF network.

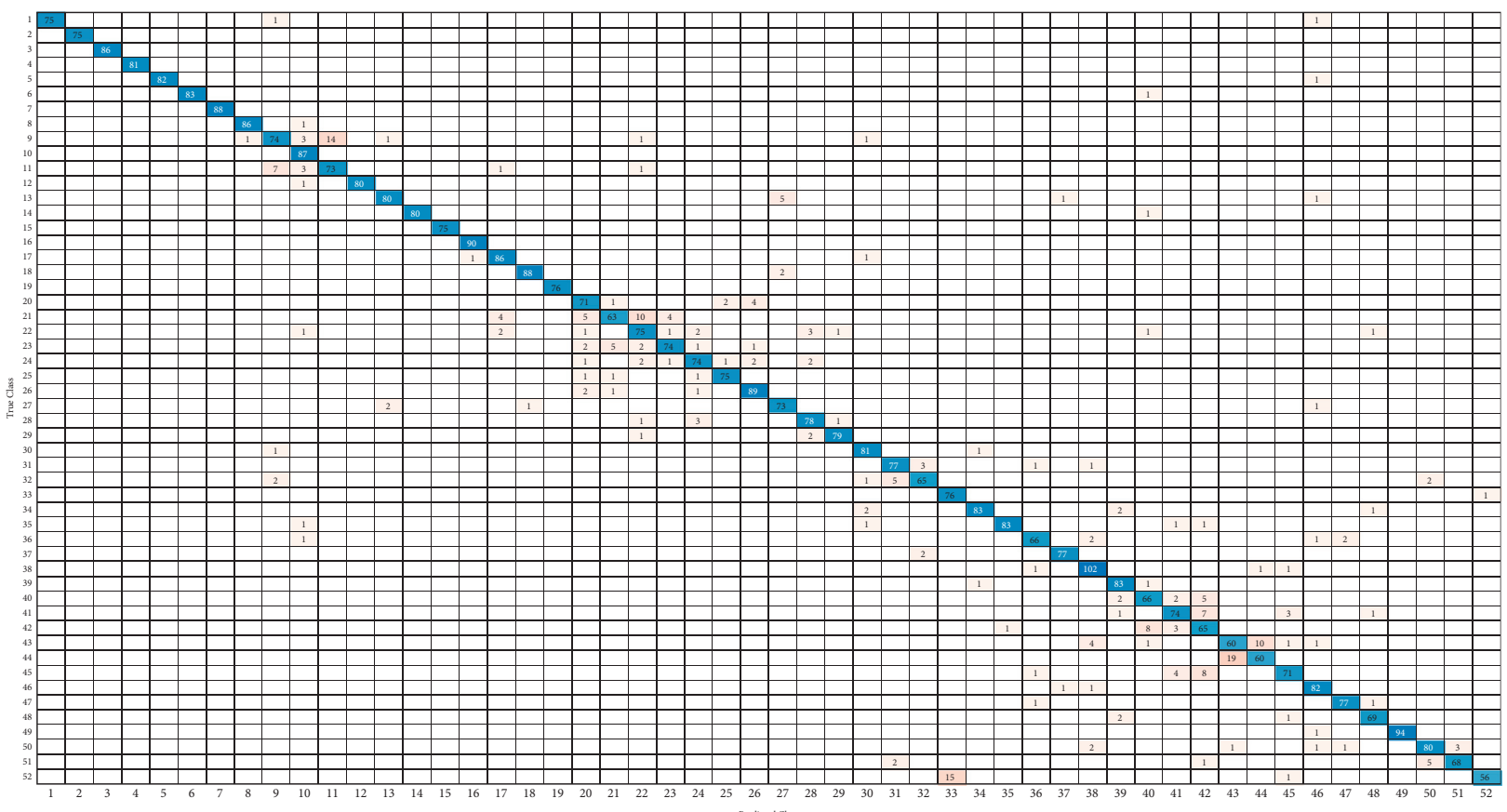

FIgURE 5: The confusion matrix chart of DBDF network.

possible. A comparison of different classification networks without downsampling is shown in Table 6. In the same classification network, the validation accuracy with downsampling is higher than that without downsampling, and using downsampling saves more training time.
5.3. Computational Cost. DBDF network is the core of the proposed approach and the DBDF network is one kind of the deep learning networks. The DBDF network is adapted to use a GPU accelerated approach to gain a significant boost in performance and GPU will shorten the training time of the 
TABle 3: The accuracy for 52 movements.

\begin{tabular}{|c|c|c|c|c|c|c|c|}
\hline Label & Accuracy (\%) & Label & Accuracy (\%) & Label & Accuracy (\%) & Label & Accuracy (\%) \\
\hline 2 & 100.0 & 14 & 98.8 & 48 & 95.8 & 40 & 88.0 \\
\hline 3 & 100.0 & 33 & 98.7 & 26 & 95.7 & 23 & 87.1 \\
\hline 4 & 100.0 & 18 & 97.8 & 35 & 95.4 & 32 & 86.7 \\
\hline 7 & 100.0 & 17 & 97.7 & 27 & 94.8 & 41 & 86.0 \\
\hline 10 & 100.0 & 39 & 97.6 & 34 & 94.3 & 11 & 85.9 \\
\hline 15 & 100.0 & 46 & 97.6 & 28 & 94.0 & 22 & 85.2 \\
\hline 16 & 100.0 & 30 & 97.6 & 31 & 93.9 & 45 & 84.5 \\
\hline 19 & 100.0 & 37 & 97.5 & 13 & 92.0 & 42 & 84.4 \\
\hline 49 & 98.9 & 47 & 97.5 & 36 & 91.7 & 43 & 77.9 \\
\hline 8 & 98.9 & 1 & 97.4 & 20 & 91.0 & 9 & 77.9 \\
\hline 6 & 98.8 & 38 & 97.1 & 50 & 90.9 & 52 & 77.8 \\
\hline 5 & 98.8 & 29 & 96.3 & 51 & 89.5 & 44 & 75.9 \\
\hline 12 & 98.8 & 25 & 96.2 & 24 & 89.2 & 21 & 73.3 \\
\hline
\end{tabular}

TABLE 4: Comparison of the number of movements and classification accuracy of the proposed method with existing algorithms.

\begin{tabular}{|c|c|c|c|c|}
\hline Author & Classification algorithm & Sensing material & Number of movements & $\begin{array}{c}\text { Accuracy of } \\
\text { classification (\%) }\end{array}$ \\
\hline Nassour et al. [1] & A linear regression & Electrolyte solution resistance & 15 & 89.4 \\
\hline Chen et al. [2] & Support vector machine & Flexion sensors and force sensors & 16 & 89.4 \\
\hline Pan et al. [3] & Extremely randomized trees & Capacitive pressure sensors & 10 & 99.7 \\
\hline Maitre et al. [4] & Random forest & The force and bending sensors & 8 & 95 \\
\hline Lee and Bae [5] & LSTM & Eutectic gallium indium sensors & 11 & 100 \\
\hline Ayodele et al. [6] & $\mathrm{CNN}$ & Weft knit sensors & 6 & 88.27 \\
\hline Chauhan et al. [7] & Gaussian process & Rotary potentiometers & 5 & 75 \\
\hline Fatimah et al. [8] & Ensemble subspace discriminant & Surface electromyogram & 17 & 93.53 \\
\hline Maitre et al. [9] & CNN-LSTM & The force and bending sensors & 13 & 93 \\
\hline Huang et al. [10] & Dynamic time warping & RGO-coated fiber sensors & 9 & 98.3 \\
\hline Lun et al. [11] & GCNs-Net & Electroencephalogram & 4 & 96.24 \\
\hline Hou et al. [12] & BiLSTM-GCN & Electroencephalogram & 4 & 98.81 \\
\hline Proposed work & DBDF & Flexion sensors (CyberGlove II) & 52 & 93.15 \\
\hline
\end{tabular}

TABLE 5: Comparison of different classification networks.

\begin{tabular}{lcc}
\hline Classification network & Validation accuracy (\%) & Training time (min) \\
\hline Single bilstmLayer Single fullyConnectedLayer & 84.41 & 3 \\
Single bilstmLayer Double fullyConnectedLayers & 90.77 & 4 \\
Double bilstmLayers Double fullyConnectedLayers (DBDF network) & 93.15 & 5 \\
Triple bilstmLayers Double fullyConnectedLayers & 91.33 & 6 \\
Double bilstmLayers Triple fullyConnectedLayers & 91.2 & 5 \\
\hline
\end{tabular}

TABLE 6: Comparison of different classification networks without downsampling.

\begin{tabular}{lcc}
\hline Model & Validation accuracy (\%) & Training time \\
\hline Single bilstmLayer Single fullyConnectedLayer & 78.65 & $11 \mathrm{~min}$ \\
Single bilstmLayer Double fullyConnectedLayers & 88.14 & $12 \mathrm{~min}$ \\
Double bilstmLayers Double fullyConnectedLayers (DBDF network) & 89.24 & 13 min \\
Triple bilstmLayers Double fullyConnectedLayers & 87.99 & 14 min \\
Double bilstmLayers Triple fullyConnectedLayers & 89.22 & 13 min \\
\hline
\end{tabular}

DBDF network. GPU usage, GPU memory usage, GPU power usage, and training time are the key metrics of evaluating the computational cost of the proposed approach because experiments are running on a single GPU. Because training time metrics have been shown in Tables 5 and 6 , GPU usage, GPU memory usage, and GPU power usage will be discussed as follows. Data in this section are logged by GPU-Z. 


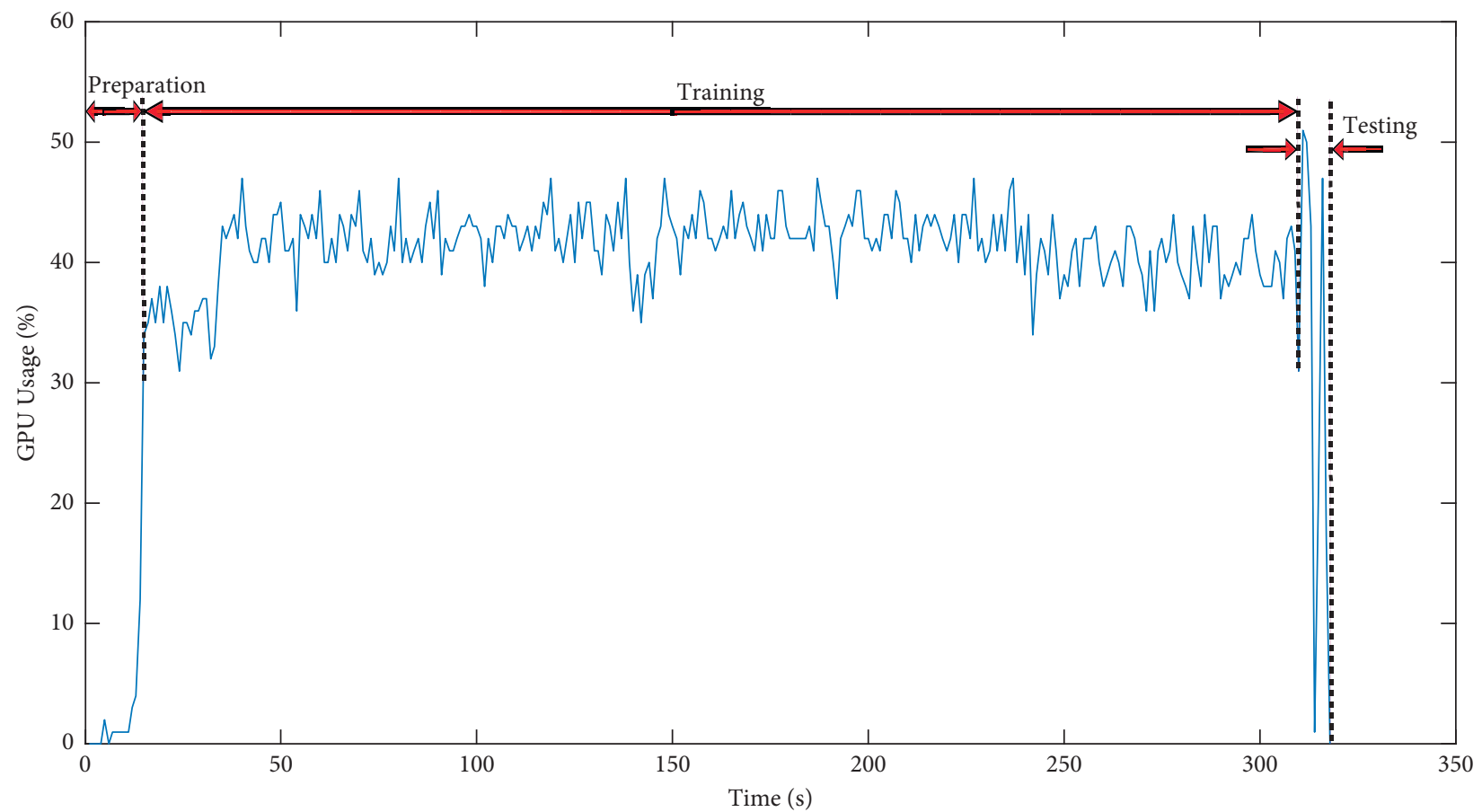

Figure 6: GPU usage trend.

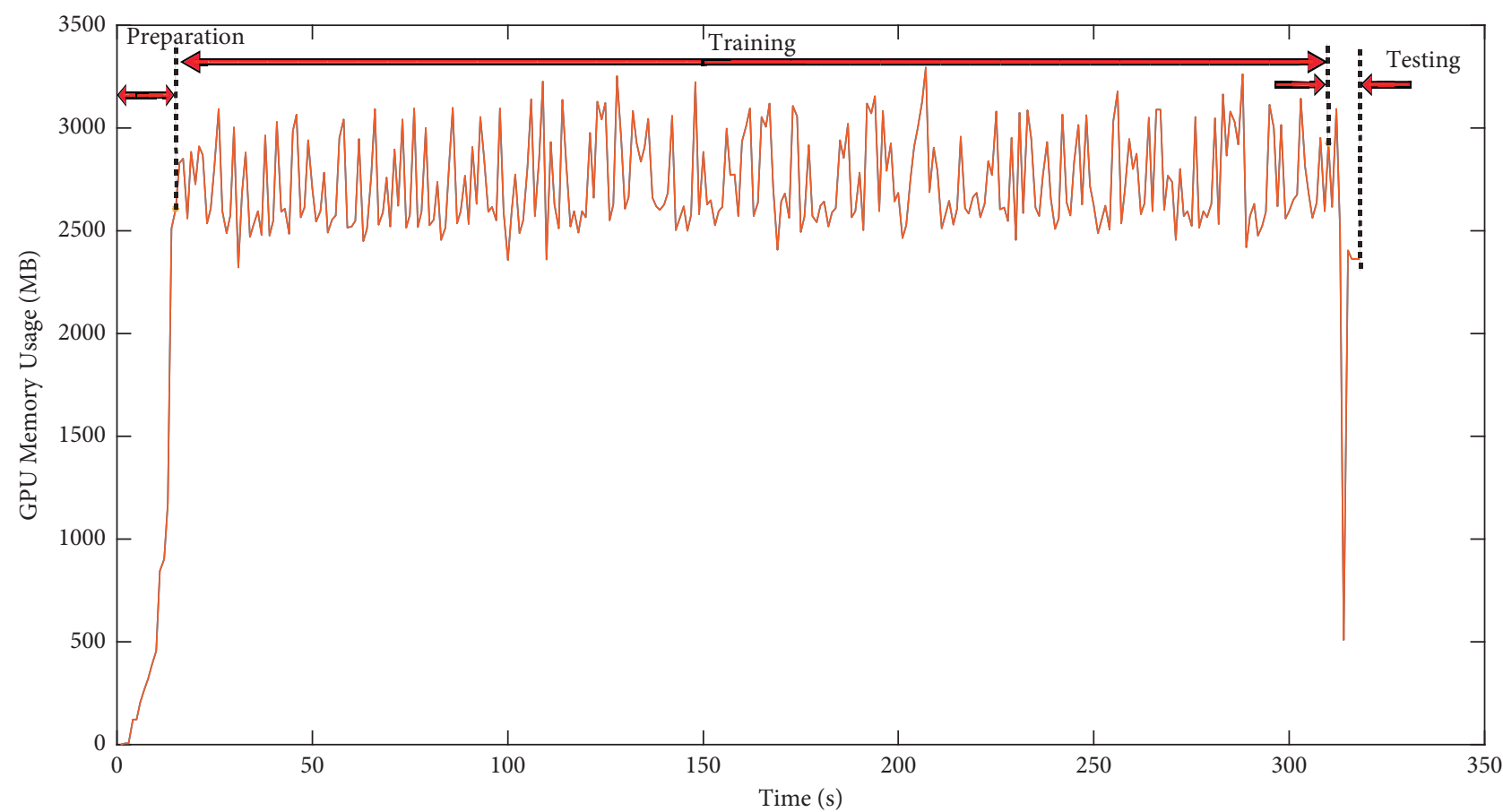

FigURE 7: GPU memory usage trend.

Figure 6 is a GPU usage trend when the movements classification algorithm is running. The preparation process means the initial run of the algorithm program. At this time, GPU usage and GPU memory usage are nearly zero. In Figure 7, the DBDF network training causes over 30\% GPU usage, and GPU memory usage increases gradually to about $2500 \mathrm{MB}$.

In Figure 8, GPU basic power consumption is $9 \mathrm{~W}$ when GPU is power on. GPU power consumption is over $60 \mathrm{~W}$ when initializing the construct of the DBDF network and is 


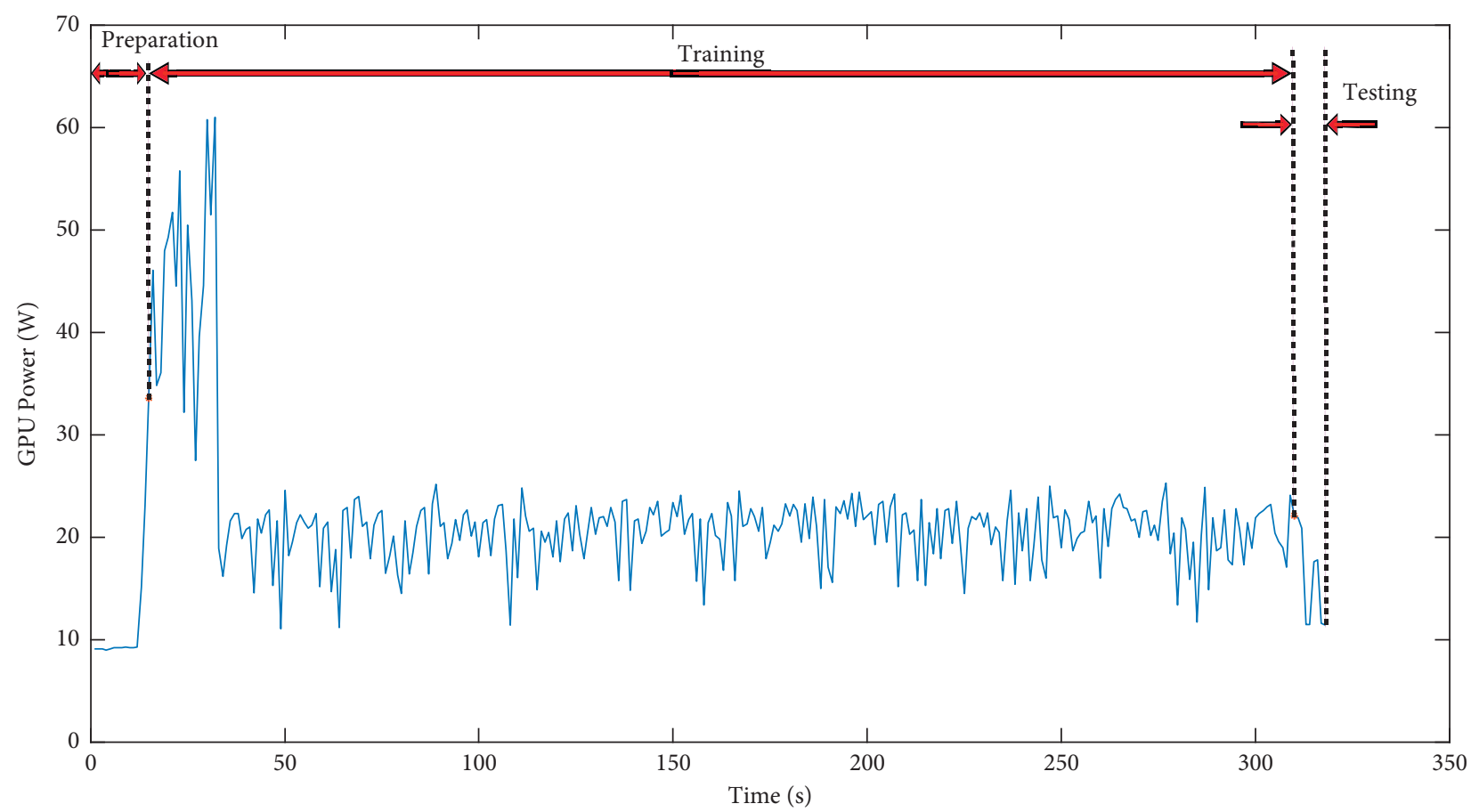

FIgURE 8: GPU power usage trend.

TABle 7: Metrics for evaluating GPU performance.

\begin{tabular}{lccc}
\hline & GPU usage (\%) & GPU memory (MB) & $\begin{array}{c}\text { GPU } \\
\text { power }(W)\end{array}$ \\
\hline Maximum & 51 & 3294 & 61 \\
Minimum & 0 & 0 & 9 \\
Mean & 39.3 & 2630.95 & 21.37 \\
\hline
\end{tabular}

average nearly $21 \mathrm{~W}$ in the program running process. In the testing process, GPU usage and GPU power consumption will decrease to $0 \%$ and $9 \mathrm{~W}$, respectively, but about $2500 \mathrm{MB}$ GPU memory is occupied.

Table 7 summarizes metrics for evaluating the GPU performance of the movements classification algorithm.

\section{Conclusions}

In this paper, we have presented the use of Fine KNN and BiLSTM network on hand movement classification using one data glove. The fine KNN classifier is the core of the movement detection algorithm, and the sensor sequences of a hand movement over a period are obtained by this algorithm. The hand movement classification algorithm includes downsampling and the DBDF network. The DBDF network architecture consists of two BiLSTM layers and two fully connected layers. Notably, the average classification accuracy of our algorithm is $93.15 \%$ in Ninapro DB1.

Because a hand is an elastic object, there are different features between the same movements and high similar features between different movements [23]. Our approach can detect 52 hand movements while just cooperating with data gloves. This approach performs better than the existing state-of-the-art algorithms in recognizing the number of hand movements and maintains high classification accuracy from 27 intact subjects. The downsampling technology is introduced to improve neural network stability and modeling performance by scaling data.

The prediction accuracies of movements 43, 9, 52, 44, 21 are lower than $80 \%$ and we will focus on improving the recognition accuracy of these movements. In the future, we will optimize the movements classification algorithm and consider more deep learning networks to improve the accuracy in Ninapro DB1 and other movement prediction applications. We are also interested in studying the DBDF network parameters transferability from one dataset to another without retraining.

\section{Data Availability}

The Ninapro DB1 data used to support the findings of this study have been deposited in the Ninapro Project repository (http://ninapro.hevs.ch/).

\section{Conflicts of Interest}

The author declares that there are no conflicts of interest regarding the publication of this study.

\section{Acknowledgments}

This work was partially supported by Guangdong University of Education Teaching Quality and Teaching Reform Project (no. 2018sfjd02) and the Appropriative Researching Fund for Guangdong Provincial Key Laboratory of Precision Equipment and Manufacturing Technology under Grant PEM201604. 


\section{References}

[1] J. Nassour, H. G. Amirabadi, S. Weheabby, A. A. Ali, H. Lang, and F. Hamker, "A robust data-driven soft sensory glove for human hand motions identification and replication," IEEE Sensors Journal, vol. 20, no. 21, pp. 12972-12979, 2020.

[2] X. Chen, L. Gong, L. Wei et al., "A wearable hand rehabilitation system with soft gloves," IEEE Transactions on Industrial Informatics, vol. 17, no. 2, pp. 943-952, 2020.

[3] J. Pan, Y. Luo, Y. Li, C.-K. Tham, C.-H. Heng, and A. V.-Y. Thean, "A wireless multi-channel capacitive sensor system for efficient glove-based gesture recognition with AI at the edge," IEEE Transactions on Circuits and Systems II: Express Briefs, vol. 67, no. 9, pp. 1624-1628, 2020.

[4] J. Maitre, C. Rendu, K. Bouchard, B. Bouchard, and S. Gaboury, "Basic daily activity recognition with a data glove," Procedia Computer Science, vol. 151, pp. 108-115, 2019.

[5] M. Lee and J. Bae, "Deep learning based real-time recognition of dynamic finger gestures using a data glove," IEEE Access, vol. 8, pp. 219923-219933, 2020.

[6] E. Ayodele, T. Bao, S. A. R. Zaidi et al., "Grasp classification with weft knit data glove using a convolutional neural network," IEEE Sensors Journal, vol. 21, no. 9, pp. 10824-10833, 2021.

[7] R. Chauhan, B. Sebastian, and P. Ben-Tzvi, "Grasp prediction toward naturalistic exoskeleton glove control," IEEE transactions on human-machine systems, vol. 50, no. 1, pp. 22-31, 2019.

[8] B. Fatimah, P. Singh, A. Singhal, and R. B. Pachori, "Hand movement recognition from sEMG signals using fourier decomposition method," Biocybernetics and Biomedical Engineering, vol. 41, no. 2, pp. 690-703, 2021.

[9] J. Maitre, C. Rendu, K. Bouchard, B. Bouchard, and S. Gaboury, "Object recognition in performed basic daily activities with a handcrafted data glove prototype," Pattern Recognition Letters, vol. 147, pp. 181-188, 2021.

[10] X. a. Huang, Q. Wang, S. Zang et al., "Tracing the motion of finger joints for gesture recognition via sewing RGO-coated fibers onto a textile glove," IEEE Sensors Journal, vol. 19, no. 20, pp. 9504-9511, 2019.

[11] X. Lun, S. Jia, Y. Hou, Y. Shi, and Y. Li, "GCNs-net: a graph convolutional neural network approach for decoding timeresolved eeg motor imagery signals," Signal Processing, vol. 1 https://ui.adsabs.harvard.edu/abs/2020arXiv200608924L.

[12] Y. Hou, S. Jia, X. Lun, Y. Shi, and Y. Li, “Deep feature mining via attention-based BiLSTM-GCN for human motor imagery recognition," Signal Processing, vol. 1 https://ui.adsabs. harvard.edu/abs/2020arXiv200500777H.

[13] Y. Hou, L. Zhou, S. Jia, and X. Lun, "A novel approach of decoding EEG four-class motor imagery tasks via scout ESI and CNN," Journal of Neural Engineering, vol. 17, no. 1, Article ID 016048, 2020.

[14] X. Tang, Y. Liu, C. Lv, and D. Sun, "Hand motion classification using a multi-channel surface electromyography sensor," Sensors, vol. 12, no. 2, pp. 1130-1147, 2012, https://www. mdpi.com/1424-8220/12/2/1130.

[15] M. Atzori, A. Gijsberts, I. Kuzborskij et al., "Characterization of a benchmark database for myoelectric movement classification," IEEE Transactions on Neural Systems and Rehabilitation Engineering, vol. 23, no. 1, pp. 73-83, 2015.

[16] M. Atzori, A. Gijsberts, C. Castellini et al., "Electromyography data for non-invasive naturally-controlled robotic hand prostheses," Scientific Data, vol. 1, Article ID 140053, 2014.
[17] A. Hamoudzadeh and S. Behzadi, "Predicting user's next location using machine learning algorithms," Spatial Information Research, vol. 29, no. 3, pp. 379-387, 2021.

[18] C. Iwendi, K. Mahboob, Z. Khalid, A. R. Javed, M. Rizwan, and U. Ghosh, "Classification of COVID-19 individuals using adaptive neuro-fuzzy inference system," Multimedia Systems, pp. 1-15, 2021.

[19] G. Yuan, X. Liu, Q. Yan, S. Qiao, Z. Wang, and L. Yuan, "Hand gesture recognition using deep feature fusion network based on wearable sensors," IEEE Sensors Journalournal, vol. 21, no. 1, pp. 539-547, 2020.

[20] W. Yan, H. Zhang, J. Sui, and D. Shen, "Deep chronnectome learning via full bidirectional long short-term memory networks for MCI diagnosis," in Proceedings of the Medical Image Computing and Computer Assisted Intervention - MICCAI 2018, vol. 11072, pp. 249-257, Granada, Spain, September 2018.

[21] X. Yan, T. Guan, K. Fan, and Q. Sun, "Novel double layer bilstm minor soft fault detection for sensors in air-conditioning system with KPCA reducing dimensions," Journal of Building Engineering, vol. 44, Article ID 102950, 2021.

[22] C. Zhao, X. Huang, Y. Li, and M. Yousaf Iqbal, "A doublechannel hybrid deep neural network based on CNN and BiLSTM for remaining useful life prediction," Sensors, vol. 20, no. 24, Article ID 7109, 2020.

[23] Y. Xu and Y. Dai, "Review of hand gesture recognition study and application," Contemporary Engineering Sciences, vol. 10, no. 8, pp. 375-384, 2017. 